\title{
Acupuncture treatment for insulin sensitivity of women with polycystic ovary syndrome and insulin resistance: a study protocol for a randomized controlled trial
}

Juan $\mathrm{Li}^{1}$, Ernest Hung Yu Ng${ }^{2}$, Elisabet Stener-Victorin ${ }^{3}$, Zhenxing Hu${ }^{4}$, Xiaoguang Shao ${ }^{5}$, Haiyan Wang ${ }^{5}$, Meifang $\mathrm{Li}^{1}$, Maohua Lai ${ }^{1}$, Changcai Xie ${ }^{6}$, Nianjun $\mathrm{Su}^{7}$, Chuyi $\mathrm{Yu}^{1}$, Jia Liu', Taixiang Wu ${ }^{8}$ and Hongxia Ma ${ }^{1 *}$

\begin{abstract}
Background: Our prospective pilot study of acupuncture affecting insulin sensitivity on polycystic ovary syndrome (PCOS) combined with insulin resistance (IR) showed that acupuncture had a significant effect on improving the insulin sensitivity of PCOS. But there is still no randomized controlled trial to determine the effect of acupuncture on the insulin sensitivity in women with PCOS and IR. In this article, we present the protocol of a randomized controlled trial to compare the effect of true acupuncture on the insulin sensitivity of these patients compared with metformin and sham acupuncture. Acupuncture may be an effective therapeutic alternative that is superior to metformin and sham acupuncture in improving the insulin sensitivity of PCOS combined with IR.

Methods: This study is a multi-center, controlled, double-blind, and randomized clinical trial aiming to evaluate the effect of acupuncture on the insulin sensitivity in PCOS combined with IR. In total 342 patients diagnosed with PCOS and IR will be enrolled. Participants will be randomized to one of the three groups: (1) true acupuncture + metformin placebo; (2) sham acupuncture + metformin, and (3) sham acupuncture + metformin placebo. Participants and assessors will be blinded. The acupuncture intervention will be given 3 days per week for a total of 48 treatment sessions during 4 months. Metformin ( $0.5 \mathrm{~g}$ per pill) or placebo will be given, three times per day, and for 4 months. Primary outcome measures are changes in homeostasis model assessment of insulin resistance (HOMA-IR) and improvement rate of HOMA-IR by oral glucose tolerance test (OGTT) and insulin releasing test (Ins). Secondary outcome measures are homeostasis model assessment $\beta$ (HOMA- $\beta$ ), area under the curve for glucose and insulin, frequency of regular menstrual cycles and ovulation, body composition, metabolic profile, hormonal profile, questionnaires, side effect profile, and expectation and credibility of treatment. Outcome measures are collected at baseline, at the end of treatments, and 3 months after the last acupuncture treatment. On completion of the screening visit, randomization will be conducted using a central randomization system.
\end{abstract}

Discussion: This study will investigate the effects of acupuncture on the insulin sensitivity of PCOS and IR women compared with metformin and sham acupuncture. We will test whether true acupuncture with needles placed in skeletal muscles and stimulated manually and by electrical stimulation is more effective than metformin and sham acupuncture with superficial needle placement with no manual or electrical stimulation in improving the insulin sensitivity in PCOS women with IR.

(Continued on next page)

\footnotetext{
* Correspondence: doctorhongxia@126.com

'Department of Traditional Chinese Medicine, the First Affiliated Hospital of

Guangzhou Medical University, Guangzhou, China

Full list of author information is available at the end of the article
}

(c) The Author(s). 2017 Open Access This article is distributed under the terms of the Creative Commons Attribution 4.0 International License (http://creativecommons.org/licenses/by/4.0/), which permits unrestricted use, distribution, and reproduction in any medium, provided you give appropriate credit to the original author(s) and the source, provide a link to the Creative Commons license, and indicate if changes were made. The Creative Commons Public Domain Dedication waiver (http://creativecommons.org/publicdomain/zero/1.0/) applies to the data made available in this article, unless otherwise stated. 
(Continued from previous page)

Trial registration: ClinicalTrials.gov, NCT02491333; Chinese Clinical Trial Registry, ChiCTR-ICR-15006639. Registered on 24 June 2015.

Keywords: Acupuncture, Metformin, Sham acupuncture, Polycystic ovary syndrome, Insulin resistance

\section{Background}

Polycystic ovary syndrome (PCOS) is one of the most common endocrine disorders in reproductive age women. It is characterized by chronic anovulation and hyperandrogenism and is often accompanied by obesity and insulin resistance (IR) as IR is present in nearly $40 \%$ of PCOS [1]. In addition, IR, hyperinsulinemia, and dyslipidemia worsen with aging, and the risk of miscarriage is three times higher than in healthy women [2]. They are also at an increased risk of pregnancy complications such as impaired glucose tolerance, gestational diabetes mellitus, pregnancy-induced hypertension and preeclampsia, and small for gestational age children [2]. It may affect general health and the quality of life. Both hyperandrogenism and IR contribute to the pathogenesis of many aspects of PCOS $[3,4]$.

IR in PCOS results from hyperinsulinemia and defects in the insulin signaling pathway. Further, high concentrations of insulin reduce circulating levels of sex hormone binding globulin (SHBG) and increase levels of free testosterone, and the latter leads to menstrual disturbance, development of ovarian cysts, hirsutism, and anovulatory infertility [5]. IR leads to a higher risk of impaired glucose, type 2 diabetes, dyslipidemia, atherosclerosis, and vascular disease [6].

The first-line treatment for overweight or obese women with PCOS is modification of diet and lifestyle. This confirmed that the benefit of improved ovulation and live birth with delayed infertility treatment with clomiphene citrate when preceded by lifestyle modification with weight loss was superior to immediate treatment [7], but for many subjects it is not easy to sustain [8]. Treatments of IR with insulin-sensitizing agents have been used in women with PCOS [9]. Metformin, an insulinsensitizing agent known to decrease hepatic glucose production via a transient inhibition of the mitochondrial respiratory-chain complex 1 and an activation of the AMP-activated protein kinase (a cellular metabolic sensor [10]), improves irregular menstrual cycles in women with PCOS whether IR is present or not [11]. Nevertheless, it is not effective in treating hirsutism, acne, or anovulatory infertility [12]. It also has side effects such as gastrointestinal irritation (diarrhea and nausea) and lactic acidosis, which is rare but serious [13]. In addition, metformin is not recommended for patients with kidney, lung, liver, and heart disease or on a low-carbohydrate diet [14]. Other common insulin-sensitizing agents are thiazolidinediones, including troglitazone (which is no longer available for clinical use), rosiglitazone, and pioglitazone [15]. However, rosiglitazone may result in cardiovascular diseases [16], and pioglitazone may increase the risk of bladder cancer [17]. Both may double the risk of bone fractures [18]. Given that current pharmacological treatments have such side effects, there is an urgent need to find a better and safer therapy for IR in PCOS women.

As an integrated part of Traditional Chinese Medicine and a relatively safe treatment [19], acupuncture treatment for PCOS has gained increasing attention in recent years. The effect of acupuncture is most likely mediated via activation of sensory nerve fibers, which in turn modulate the sympathetic activity to the ovaries and in the central nervous system [20,21]. A review found that acupuncture can correct various metabolic disorders contributing to the development of IR, such as hyperglycemia, overweight, hyperphagia, hyperlipidemia, inflammation, altered activity of the sympathetic nervous system, and insulin signal defect [22]. Thus, acupuncture may have the potential to improve insulin sensitivity [22]. Other findings suggest that electrical acupuncture stimulation affects more functional signaling pathways related to insulin sensitivity, while manual stimulation of acupuncture needles has a greater effect on glucose tolerance [23]. Furthermore, in female insulin-resistant rats both electrical and manual acupuncture stimulation enhances insulin sensitivity via activation of sensory afferents [24]. Our prospective pilot study (ClinicalTrials.gov NCT02026323) [25] of acupuncture affecting the insulin sensitivity in 81 cases of women with PCOS with IR showed that acupuncture has a significant effect on improving the insulin sensitivity in these patients. But there is still no randomized controlled trial to determine the effect of acupuncture on insulin sensitivity in women with PCOS combined with IR.

Thus, we present the protocol of a randomized controlled trial to investigate the effects of acupuncture on the insulin sensitivity of women with PCOS combined with IR. We hypothesize that true acupuncture is superior to metformin and sham acupuncture in improving insulin sensitivity.

\section{Objectives}

The primary objective of this randomized controlled trial is to evaluate the hypothesis that true acupuncture + metformin placebo (group 1) improves insulin sensitivity, as 
measured by changes in the homeostasis model assessment of insulin resistance (HOMA-IR), more effectively than sham acupuncture + metformin (group 2) and sham acupuncture + metformin placebo (group 3 ) in women with PCOS and IR. Secondary objectives include changes in HOMA- $\beta$ (islet $\beta$-cell function), C-peptide index, the insulin response to glucose calculated from the oral glucose tolerance test (OGTT) and insulin releasing test (Ins), frequency of the regular menstrual cycle and ovulation, circulating sex steroids, lipid profile, health-related quality of life and symptoms of anxiety and depression, side effects, and credibility.

\section{Methods}

\section{Overview}

This is a randomized controlled trial to determine the effect of true acupuncture + metformin placebo for the improvement of insulin sensitivity in women with PCOS and IR compared with sham acupuncture + metformin and sham acupuncture + metformin placebo. The design of this study is compliant with the Consolidated Standards of Reporting Trials (CONSORT) guidelines [26] and with the Standard Protocol Items: Recommendations for Interventional Trials (SPIRIT) statement 2013 [27] [see Additional file 1].

\section{Patients and enrollment}

Women with PCOS and IR will be recruited from the Department of Traditional Chinese Medicine in the First Affiliated Hospital of Guangzhou Medical University, Xuzhou Maternity \& Child Health Hospital, Dalian Municipal Women and Children's Medical Center, Guangdong Women and Children's Hospital, and Hexian Memorial Affiliated Hospital of Southern Medical University if they meet the inclusion criteria and do not have any exclusion criteria. Eligible subjects will be approached and sign the consent form after detailed explanation of the study design and comprehensive counseling (see the flow diagram in Fig. 1).

\section{Inclusion Criteria}

(1)Chinese women aged from 18 to 40 years.

(2) Body mass index (BMI) $\geq 18.5 \mathrm{~kg} / \mathrm{m}^{2}$.

(3) Confirmed diagnosis of PCOS according to the Rotterdam criteria in 2003 including at least two of the following three features: (1) oligo(an intermenstrual interval $>35$ days or $<8$ cycles in the past year) or amenorrhea (an intermenstrual interval >90 days), and/or (2) polycystic ovarian morphology, i.e., presence of $>12$ antral follicles $(\leq 9 \mathrm{~mm})$ and/or ovarian volume $>10 \mathrm{ml}$ on ultrasonography scanning, and/or (3) clinical and/or biochemical hyperandrogenism. Clinical hyperandrogenism on the Chinese Mainland is defined as a Ferriman-Gallwey (FG) score $\geq 5$ [28]; biochemical hyperandrogenism is total testosterone

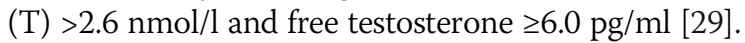

(4) Presence of IR as defined by the homeostatic model assessment-HOMA-IR: [fasting insulin $(\mu \mathrm{U} / \mathrm{ml}) \times$ fasting glucose $(\mathrm{mmol} / \mathrm{l})] / 22.5)$. A value $\geq 2.14$ is considered to be indicative of IR [30].

(5)No immediate fertility wish and willingness to use barrier contraceptive methods for 7 months.

(6) Willingness to sign the consent form.

\section{Exclusion Criteria}

(1) Exclusion of other endocrine disorders:

(1) Uncorrected thyroid disease (defined as thyroid stimulating hormone (TSH) $<0.2 \mathrm{mIU} / \mathrm{ml}$ or $>5.5 \mathrm{mIU} / \mathrm{ml}$, triiodothyronine $(\mathrm{T} 3)<1.4 \mathrm{nmol} / \mathrm{l}$ or $>2.2 \mathrm{nmol} / \mathrm{l}$, and free thyroxine (T4) $<10$ $\mathrm{pmol} / \mathrm{l}$ or $>23 \mathrm{pmol} / \mathrm{l})$. A normal level within the last year is adequate for entry.

(2) Poorly controlled type I or type II diabetes (defined as a glycosylated hemoglobin (HbA1c) level $>7.0 \%$ ) or patients receiving antidiabetic medications such as insulin, thiazolidinediones, acarbose, or sulfonylureas likely to confound the effects of the study medication; patients currently receiving metformin XR (extended release) for a diagnosis of type I or type II diabetes or for PCOS are also excluded.

(3)Cushing's syndrome (defined as an archetype of metabolism syndrome. High glucocorticoid levels lead to muscle, liver, and adipocyte insulin resistance; 17-hydorxycorticosteroids $>55 \mathrm{umol} /$ $24 \mathrm{~h}$ or urinary-free cortisol $>304 \mathrm{nmol} / 24 \mathrm{~h}$ ).

(4)Congenital adrenal hyperplasia (defined as patients with known 21-hydroxylasedeficiency or other enzyme deficiency leading to the phenotype of congenital adrenal hyperplasia; 17 -oh progesterone $>10 \mathrm{ng} / \mathrm{ml}$ in adrenocorticotropic hormone 1$24 \mathrm{~h}$ excited test (after $60 \mathrm{~min}$ ).

(5)Suspected androgen-secreting adrenal or ovarian tumor.

(2) Use of hormonal or other medication including Chinese herbal prescriptions, which may affect the outcome of the last 2 months.

(3) Receiving acupuncture in the past 2 months.

(4) Within 6 weeks of pregnancy.

(5) Post-abortion or postpartum within the past 6 weeks.

(6) Breastfeeding within the last 4 months.

(7) Not willing to give written consent to the study.

(8) Having a bariatric surgery procedure within the past 12 months or being in a period of acute weight loss.

(9) Additional exclusion criteria include:

a. Patients on oral contraceptives, depot progestin, or hormonal implants (including Implanon). A 


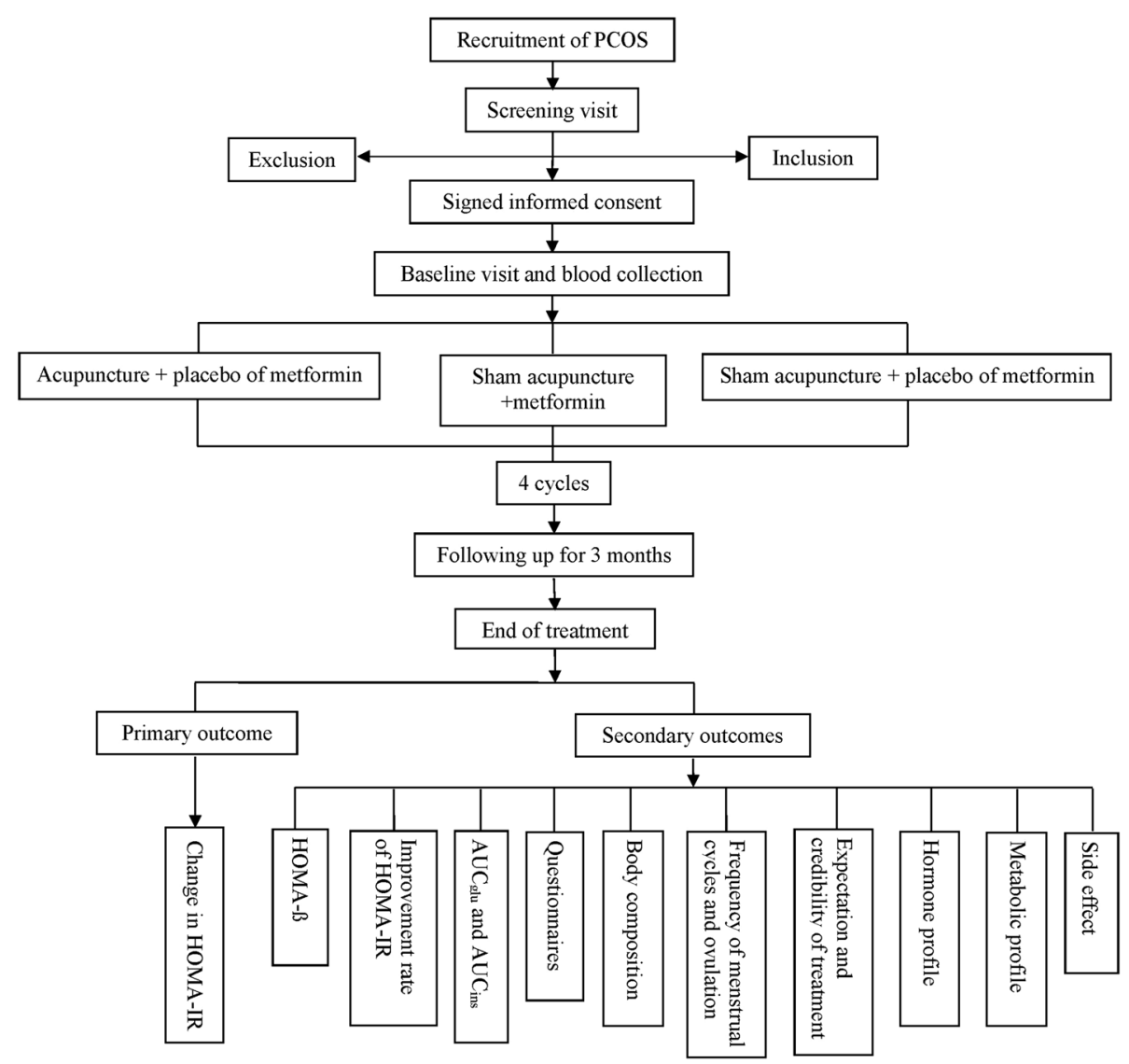

Fig. 1 CONSORT flow diagram of the study. PCOS polycystic ovary syndrome; HOMA homeostatic model assessment; IR insulin resistance; AUC area under the curve

2-month washout period will be required prior to screening for patients on these agents. Longer washouts may be necessary for certain depot contraceptive forms or implants, especially where the implants are still in place. A 1-month washout will be required for patients on oral cyclic progestin.

b. Heart disease.

c. Patients with a history of or suspected cervical carcinoma, endometrial carcinoma, or breast carcinoma.

d. Patients enrolled in other studies that require medications.

e. Patients taking longer than a 1-month break during the protocol should not be enrolled.

\section{Intervention arms}

\section{Information to all study subjects}

All participants will be advised about the importance of regular physical exercise and a balanced diet by a trained dietician before they receive the treatments. Importantly, they are instructed not to change their exercise or diet habits during the entire study period.
True acupuncture or sham acupuncture and metformin will be started 2 days after the baseline visit including OGTT and Ins. All subjects will be asked to use a barrier method for contraception. True and sham acupuncture treatment will be given three times per week. Each treatment session lasts for $30 \mathrm{~min}$ and can be separated by an interval of 1-3 days, with a maximum of 48 treatment sessions during 4 months. After the third treatment, all patients fill in the expectation and credibility questionnaire [31]. Metformin (0.5 g per pill) or placebo will be given, three times per day for 4 months.

\section{True acupuncture protocol}

The rationale of acupuncture protocols is based on traditional Chinese and Western medical theories, and the study protocol follows the CONSORT [26] and Standards for Reporting Interventions in Clinical Trials of Acupuncture (STRICTA) [32] recommendations. We will use fixed acupuncture protocols. The acupuncture protocol of this randomized controlled trial (RCT) follows the protocol in the previous RCT in women with PCOS including Acupuncture to Treat Insulin Resistance in Women With and 
Without Polycystic Ovary Syndrome (ClinicalTrials.gov NCT01457209) [33], Acupuncture and Clomiphene Citrate on Live Birth in Anovulatory Women With Polycystic Ovary Syndrome (ClinicalTrials.govNCT01573858) [34], and a prospective pilot study of the Effect of Acupuncture on Insulin Sensitivity Polycystic Ovary Syndrome (ClinicalTrials.gov NCT02026323) [25].

Disposable, single-use, sterilized needles made of stainless steel, $0.25 \times 30 \mathrm{~mm}$ and $0.30 \times 40 / 50 \mathrm{~mm}$ (Huanqiu, Suzhou Huanqiu Acupuncture Medical Instrument Co., Ltd. 215002 Suzhou, China), will be inserted to a depth of $15-35 \mathrm{~mm}$ in segmental acupuncture points located in abdominal and leg muscles with innervations corresponding to the ovaries. Two sets of acupuncture points will be alternated every second treatment (Table 1 [25]). The first set consists of the conception vessel (CV) 3, CV 12, and stomach (ST) 29 bilaterally and in the muscles above the knee, ST34, and ST 33 bilaterally and below the knee, spleen (SP) 6, and ST 36. Needles will also be placed in extra-segmental acupuncture points that do not innervate the ovaries large intestine (LI) 4 bilaterally. In total, 14 needles will be placed, and all will be stimulated manually by rotating the needle to evoke needle sensation (de $q i$ ) once when inserted. The following points will be connected to an electrical stimulator (Export Abteilung, Schwa-Medico GmbH, Wetzlarer Str. 41-43; 35630 Ehringshausen): CV 3 to CV 12, ST 29 bilateral, and ST 34 to ST 33 bilateral. Stimulations are

Table 1 Acupuncture protocol [25]

\begin{tabular}{|c|c|c|c|c|}
\hline Point & Stimulation & location & Muscle & Innervation \\
\hline \multicolumn{5}{|l|}{ Set 1} \\
\hline Zhongji (CV3) & EA & 4 cun caudal to the umbilicus & Fibrous tissue, linea alba & L1 \\
\hline Zhongwan (CV12) & EA & On the midline, 4 cun superior to the umbilicus & Fibrous tissue, linea alba & Th7-8 \\
\hline Guilai (bilateral) (ST29) & EA & $\begin{array}{l}1 \text { cun cranial to the pubic bone and } 2 \text { cun lateral } \\
\text { of the midline }\end{array}$ & M. rectus abdominis & Th6-12 \\
\hline Liangqiu (bilateral) (ST34) & EA & $\begin{array}{l}2 \text { cun above the superior lateral border of the } \\
\text { patella on the line connecting the anterior } \\
\text { superior iliac spine found with the knee flexed }\end{array}$ & M. quadriceps femoris & $\begin{array}{l}\text { Femoral } \\
\text { nerve }\end{array}$ \\
\hline Yinshi (bilateral) (ST33) & EA & $\begin{array}{l}3 \text { cun above the superior lateral border of the } \\
\text { patella on the line connecting the anterior } \\
\text { superior iliac spine found with the knee flexed }\end{array}$ & M. quadriceps femoris & $\begin{array}{l}\text { Femoral } \\
\text { nerve }\end{array}$ \\
\hline Sanyinjiao (bilateral) (SP6) & $\begin{array}{l}\text { De Qi four } \\
\text { times }\end{array}$ & 3 cun proximal to the medial malleolus & $\begin{array}{l}\text { Mm. flexor digitorum longus, tibialis } \\
\text { posterior }\end{array}$ & $\llcorner 4-5, S 1-2$ \\
\hline Zusanli (bilateral) (ST36) & $\begin{array}{l}\text { De Qi four } \\
\text { times }\end{array}$ & $\begin{array}{l}\text { On the anterior lateral side of the leg, } 3 \text { cun } \\
\text { below Dubi (ST35), one finger width } \\
\text { (middle finger) from the anterior crest of the tibia }\end{array}$ & Musculi tibialis anterior & $\llcorner 4-5, \mathrm{~S} 1$ \\
\hline Hegu (bilateral) (LI4) & $\begin{array}{l}\text { De Qi four } \\
\text { times }\end{array}$ & On the highest point at $\mathrm{m}$. interosseus dorsalis & $\begin{array}{l}\text { Mm. interosseus dorsalis I, lumbricalis } \\
\text { II, adductor pollicis }\end{array}$ & C8, Th1 \\
\hline \multicolumn{5}{|l|}{ Set 2} \\
\hline Daju (bilateral) (ST27) & EA & $\begin{array}{l}3 \text { cun cranial to the pubic bone and } 2 \text { cun } \\
\text { lateral to the midline }\end{array}$ & M. rectus abdominis & Th6-12 \\
\hline Qihai (CV6) & EA & 1.5 cun caudal to the umbilicus & Fibrous tissue, linea alba & Th11 \\
\hline Xiawan (CV10) & EA & 2 cun cranial to the umbilicus & Fibrous tissue, linea alba & Th8 \\
\hline Extra-meridian point (bilateral) & EA & 6 cun above the patella in line with SP10 & M. quadriceps femoris & L2-L4 \\
\hline Xuehai (bilateral) (SP10) & EA & $\begin{array}{l}\text { With the knee flexed, on the medial side of } \\
\text { the thigh } 2 \text { cun above the superior medial } \\
\text { corner of the patella on the prominence of } \\
\text { the medial head of the quadriceps } \\
\text { muscle of the thigh }\end{array}$ & M. quadriceps femoris & L2-L4 \\
\hline Sanyinjiao (bilateral) (SP6) & $\begin{array}{l}\text { De Qi four } \\
\text { times }\end{array}$ & 3 cun proximal to the medial malleolus & $\begin{array}{l}\text { Mm. flexor digitorum longus, tibialis } \\
\text { posterior }\end{array}$ & $\llcorner 4-5, S 1-2$ \\
\hline Taichong (bilateral) (LR3) & $\begin{array}{l}\text { De Qi four } \\
\text { times }\end{array}$ & $\begin{array}{l}\text { Between metatarsal I \& II, just distal to } \\
\text { the caput }\end{array}$ & M. interosseus dorsalis I & S2-3 \\
\hline Neiguan (bilateral) (PC6) & $\begin{array}{l}\text { De Qi four } \\
\text { times }\end{array}$ & $\begin{array}{l}2 \text { cun proximal to the processus styloideus } \\
\text { radii, between the tendons of the palmaris } \\
\text { longus and the flexor carpi radialis }\end{array}$ & M. flexor digitorum superficialis & C8, Th1 \\
\hline
\end{tabular}

The two sets will be alternated for every other treatment

$C$ cervical vertebra, $C V$ conception vessel, $E A$ electroacupuncture, $L$ lumbar vertebra, $L /$ large intestine, $L R$ liver, $M$. musculi, $M m$. musculus, $P C$ pericardium, $S$ sacral vertebra, SP spleen, ST stomach, Th thoracic vertebra 
given as low-frequency electric acupuncture (EA) of $2 \mathrm{~Hz}, 0.3 \mathrm{~ms}$ pulse length, with an intensity adjusted to produce local muscle contractions without pain or discomfort. Needles not connected to the electrical stimulator will be manually stimulated to evoke needle sensation every $10 \mathrm{~min}$, in total four times. The second set consists of 14 needles placed in segmental abdominal points and stimulated electrically: ST 27 bilaterally, CV 6 connected to CV 10; leg points: SP10 connected to a non-acupuncture point located 6 cun proximal to the patella as the medial border (electrical stimulation); SP 6 and liver (LR) 3 bilaterally (manual stimulation). Extrasegmental points are pericardium (PC) 6 bilaterally (manual stimulation).

\section{Needle insertion technique}

Needle insertion should be gentle. The skin is tightened by pressing around the area of needle insertion, and the needle is then gently inserted.

\section{Needles and stimulation}

Needle size: $0.25 \times 30 \mathrm{~mm}$ or $0.30 \times 40$ or $0.30 \times 50 \mathrm{~mm}$. Select needle length with respect to the patients' BMI: $0.25 \times 30-\mathrm{mm}$ needles in women with normal BMI, $0.30 \times 40-\mathrm{mm}$ needles in overweight women, and $0.30 \times$ 50 or $75-\mathrm{mm}$ needles in obese women. Depth of insertion may vary from patient to patient. Needles are placed with a depth deep enough to reach muscle/fibrous tissue. When a needle is inserted, the needle is stimulated gently until de $q i$ (needle sensation reflecting activation of sensory afferents). As soon as de $q i$ has been reached, the needle does not hurt or cause any pain and discomfort. Manual stimulation of needles that are not attached to an electrical stimulator is stimulated when inserted, after $10 \mathrm{~min}, 20 \mathrm{~min}$, and immediately before they are removed after $30 \mathrm{~min}$.

The electrodes are attached to an electric stimulation according to the protocol. The electric stimulator is turned on to program 10, and the intensity is gradually increased. The intensity will be as high as possible without causing pain or discomfort of subjects. The stimulation amplitude/intensity will be adjusted after 10 and $20 \mathrm{~min}$ at the same time as when manually stimulated needles are manipulated. After $30 \mathrm{~min}$ the stimulator is turned off, electrodes are disconnected, and needles are taken out.

\section{Sham acupuncture protocol}

Disposable, single-use, sterilized needles made of stainless steel, $0.20 \times 20 \mathrm{~mm}$ (Huanqiu, Suzhou Huanqiu Acupuncture Medical Instrument Co., Ltd. 215002 Suzhou, China) will be inserted superficially to a depth of $<5 \mathrm{~mm}$, one in each shoulder and one in each upper arm at nonacupuncture points (Table 2 [34]) with no stimulation. Placement of needles is unlikely to affect ovulation and IR in women with PCOS. Electrodes will be attached to the needles, and the stimulator will be turned on at an intensity of zero (no active current) to mimic EA in the acupuncture protocol. No manual stimulation of the needles will be performed. An intended adjustment of the intensity is made after $10 \mathrm{~min}, 20 \mathrm{~min}$, and again after $30 \mathrm{~min}$ when the stimulator is turned off and the needles removed.

\section{True and sham acupuncture treatment}

(1) Time of the day and the name of the acupuncturist when the patient receives acupuncture will be recorded.

(2) The intensity of stimulation ( $\mathrm{mA}$ ) is noted. It may vary between different electrodes. Note the range, e.g., $1.2-3.0 \mathrm{~mA}$.

(3) Note any other events that may affect the treatment (positive or negative).

(4) Note concomitant medications.

(5)Collect menstrual logs at the end of every cycle.

\section{Metformin and placebo}

Metformin (Bristol-Myers Squibb Co., Shanghai, China) and placebo tablets (Jaden Pharmaceutical Co., Ltd., Guangzhou, China) will be packed and tested by a commercial pharmacy supply company (Panlongyunhai Pharmaceutical Co., Kunming, Yunnan, China) specifically for this study. Metformin or placebo will be started 2 days after the baseline visit including OGTT and Ins. Subjects will take $0.5 \mathrm{~g} /$ time, three times per day for 4 months. If the patients have adverse effects such as nausea and dizziness, the dose will be reduced to $0.5 \mathrm{~g} /$ time, two times or one time per day according to the severity.

\section{Monitoring and examination during the treatment}

All subjects will be instructed not to change their physical exercise habits or diet during the entire study. They are asked to use barrier contraception, not hormonal contraception. Physical examination will be performed monthly. Every menstruation should be recorded,

Table 2 Sham acupuncture protocol [34]

\begin{tabular}{llll}
\hline Point & Stimulation & location & Skin innervation \\
\hline No known point & Sham EA & On top of acromion & C3-4, n. supraclavicularis \\
No known point & Sham EA & On humerus, behind LI14 & C5-6, n. cutaneous brachii lateralis \\
\hline
\end{tabular}


including the date, volume, and duration of menstruation during the entire study and follow-up. The conditions of physical exercise and diet will be recorded for every cycle.

\section{Study-specific visits and procedures}

Patients will attend five visits, including the screening visit, baseline measurements, treatment visit, aftertreatment measurements, and follow-up measurements. Months indicate when each specific measurement takes place. Adverse events and concomitant medications will be recorded during every visit. The overview of study visits is shown in Table 3.

\section{Screening visit}

Women are screened in the morning after an overnight $12-\mathrm{h}$ fast. Detailed information about the study design is given.

\section{Obtain signed informed consent}

Complete physical examination Complete physical examinations will be performed including height, weight, hip, and waist measurements. Height and weight will be recorded to the nearest 0.1 cun and $0.1 \mathrm{~kg}$, respectively. Waist and hip circumference will be recorded to the nearest 1 cun. Hirsute assessment by FG, acne standard acne lesion counts, and pelvic examination will be conducted.

\section{Perform transvaginal or transrectal ultrasound of ovaries}

Ovaries, including the ovarian size in three dimensions, the size of the largest ovarian follicle/cyst and size of every follicle with a mean diameter greater than $10 \mathrm{~mm}$, and total antral follicle count (small follicles with mean diameter $<10 \mathrm{~mm}$ ) of each ovary will be obtained through transvaginal or transrectal ultrasound.

\section{Check urine pregnancy test}

Fasting blood samples to exclude other endocrine disorders Fasting (at least $4 \mathrm{~h}$ ) blood samples are taken to exclude any endocrine disorders:

Fasting glucose, fasting insulin, $\mathrm{HbA} 1 \mathrm{C}(>7 \%), \mathrm{C}$ peptide, TSH $(<0.2 \mathrm{mIU} / \mathrm{ml}$ or $>5.5 \mathrm{mIU} / \mathrm{ml}), \mathrm{T} 3$ $(<1.4 \mathrm{nmol} / \mathrm{l}$ or $>2.2 \mathrm{nmol} / \mathrm{l})$, and free T4 $(<10 \mathrm{pmol} / \mathrm{l}$ or $>23 \mathrm{pmol} / \mathrm{l})$.

\section{Progestin withdrawal}

Provide progestin prescription to induce withdrawal bleeding, with instructions to begin medication once eligibility has been determined.

\section{Baseline visit}

After the screening visit, if a woman fulfills inclusion criteria and has signed the informed consent form, she will be assigned to the baseline visit. The baseline visit takes place day $2-5$ of a spontaneous period or after a withdrawal bleeding after an overnight fast.

\section{Laboratory Examination}

Blood will be collected. Part of it will be stored, and the remainder is used for determination of DNA and sphingolipid levels.

(1) Circulating sex steroids: Fasting serum levels of follicle-stimulating hormone (FSH), luteinizing hormone (LH), estradiol (E2), prolactin (PRL) and $\mathrm{T}$, and sex hormone binding globulin (SHBG).

Table 3 Overview of study visits

\begin{tabular}{|c|c|c|c|c|c|c|c|c|}
\hline & Screening visit & Baseline visit & Mor & & & & & \\
\hline & & & $1 \mathrm{st}$ & 2nd & $3 \mathrm{rd}$ & 4 th & $\begin{array}{l}4 \text { months } \\
\text { of treatment }\end{array}$ & $\begin{array}{l}\text { visit } 3 \text { months after } \\
\text { the last acupuncture } \\
\text { treatment }\end{array}$ \\
\hline OGTT and Ins & & $\sqrt{ }$ & & & & & $\sqrt{ }$ & $\sqrt{ }$ \\
\hline $\begin{array}{l}\text { Body composition (weight, height, waist circumference, } \\
\text { hip circumference, FG/acne) }\end{array}$ & $\sqrt{ }$ & $\sqrt{ }$ & $\sqrt{ }$ & $\sqrt{ }$ & $\sqrt{ }$ & $\sqrt{ }$ & $\sqrt{ }$ & $\sqrt{ }$ \\
\hline Menstrual cycle diary & $\sqrt{ }$ & $\sqrt{ }$ & $\sqrt{ }$ & $\sqrt{ }$ & $\sqrt{ }$ & $\sqrt{ }$ & $\sqrt{ }$ & $\sqrt{ }$ \\
\hline $\begin{array}{l}\text { Fasting blood samples for FGLU, FINS, HbA1c, C-peptide, } \\
\text { TSH, T3, free T4 }\end{array}$ & $\sqrt{ }$ & & & & & & & \\
\hline Fasting blood samples for FSH, LH, SHBG, T, E2, PRL & & $\sqrt{ }$ & & & & & $\sqrt{ }$ & $\sqrt{ }$ \\
\hline $\begin{array}{l}\text { Fasting blood samples for ApoA1,ApoB, TC, TG, HDL-C, } \\
\text { LDL-C, BRT, renal and liver profile }\end{array}$ & & $\sqrt{ }$ & & & & & $\sqrt{ }$ & $\sqrt{ }$ \\
\hline Transvaginal or transrectal ultrasound & $\sqrt{ }$ & & & & & & $\sqrt{ }$ & $\sqrt{ }$ \\
\hline Questionnaires & & $\sqrt{ }$ & & & & & $\sqrt{ }$ & $\sqrt{ }$ \\
\hline
\end{tabular}

Apoa1 apolipoprotein A1, ApoB apolipoprotein B, BRT blood routine test, E2 estradiol, FG Ferriman-Gallwey score, FGLU fasting blood glucose, FINS Fasting insulin, $F S H$ follicle-stimulating hormone, $H b A 1 c$ glycosylated hemoglobin, $H D L-C$ high density lipoprotein, $L D L-C$ low density lipoprotein, $L H$ luteinizing hormone, $P R L$ prolactin, SHBG sex hormone-binding globulin, $T$ total testosterone, $T C$ total cholesterol, $T G$ triglyceride, $T S H$ thyroid stimulating hormone, $T 3$ triiodothyronine, $T 4$ thyroxine, OGTT oral glucose tolerance test, Ins insulin-releasing test 
(2)Lipid profile: Triglycerides (TG), total cholesterol (TC), high-density lipoprotein (HDL-C), low-density lipoprotein (LDL-C), apolipoprotein A1 (ApoA1), apolipoprotein $B(A p o B)$, blood routine test(BRT), and renal and liver profiles.

(3) Glucose homeostasis and insulin sensitivity: The OGTT and Ins with $75 \mathrm{~g}$ glucose. Blood samples will be obtained to measure plasma glucose, serum insulin, and C-peptide at 0, 60, and $120 \mathrm{~min}$ during the OGTT and Ins.

\section{Complete questionnaires}

Quality of life will be assessed by the short form 36 (SF36) [35], the Chinese Quality of Life (ChiQOL) [36], sleeping questionnaires [37], international physical activity questionnaire (IPAQ) [38], questionnaire of eating and weight patterns (QEWP) [39], and the Polycystic Ovary Syndrome Questionnaire (PCOS-QOL) [40]. We will also assess symptoms of anxiety and depression by the Zung SAS and Zung SDS questionnaires and complete the quantization table of traditional Chinese medicine (TCM) syndromes about PCOS.

\section{Assess TCM syndromes of patients}

Syndrome differentiation (Bian Zheng) in TCM is the comprehensive analysis of clinical information gained by the four main diagnostic TCM procedures: observation, listening, questioning, and pulse analysis, and it is used to guide the choice of treatment by acupuncture and/ or TCM herbal formulae [41]. In PCOS, patients are empirically differentiated into four categories: (1) phlegmdampness syndrome, (2) blood stasis syndrome, (3) phlegm, and (4) blood stasis.

\section{Visit after 4 months of acupuncture treatment}

During the end of treatment visit, all baseline measures will be repeated as listed below:

(1)Physical examination will be performed, including vital signs, height, weight, and hip and waist measurements as well as repeating hirsute and acne assessments after the end of treatment or pregnancy.

(2) The serum levels of sex hormone steroids and the metabolic profile will be repeated.

(3) The transvaginal or transrectal ultrasound will be repeated for antral follicle counts.

(4) OGTT and Ins will be repeated.

(5) Blood will be collected for storage and for determination of DNA and sphingolipid levels.

(6) QOL, sleeping, IPAQ, anxiety/depression questionnaires, QEWP, and the quantization table of TCM will be repeated.

(7)Menstrual logs will be collected.
(8)Adverse events and concomitant medications will be recorded.

(9) The questionnaires about acupuncture treatment will be answered.

\section{End of treatment visit}

The end of treatment visit will be done 3 months after the last acupuncture treatment (see points 1-8 under Visit after 4 months of acupuncture treatment). All the subjects will be followed up by visiting the acupuncturists monthly for 3 months. The conditions of physical exercise and diet will be record every month.

\section{Safety analysis}

Adverse events will be categorized and the percentage of patients experiencing adverse events and serious adverse events during the treatment period and follow-up period will be documented and reported to the Data and Safety Monitoring Board (DSMB). These will be reviewed on a quarterly basis by the DSMB, and serious adverse events will be immediately reviewed.

\section{Outcome measures}

Primary outcome

Change in HOMA-IR.

\section{Secondary outcomes}

(1)Improvement rate of HOMA-IR.

(2)HOMA- $\beta$ : Islet $\beta$-cell function will be evaluated by the formula $(20 \times$ fasting insulin $(\mathrm{mU} / \mathrm{ml}) /$ (fasting plasma glucose $(\mathrm{mmol} / \mathrm{l})-3.5))[42]$ and by the C-peptide index (CPI) Fasting C-peptide (nmol/l)/fglucose $(\mathrm{mmol} / \mathrm{l}) \times 100$ [43].

(3) The insulin response to glucose will be assessed by calculating the area under the curve during the OGTT and Ins performance for glucose (AUCglu) and insulin (AUCins) using the trapezoidal rule [44].

(4) Frequency of menstrual cycles and ovulation.

(5)Body composition: weight, BMI, waist-to-hip circumference, and FG and acne lesion counts.

(6) Metabolic profile: glucose and insulin concentrations, C-peptide, HbA1c, TC, TG, HDL-C and LDL-C, and ApoA1 and ApoB.

(7)Hormonal profile: FSH, LH, T, and SHBG.

(8)Questionnaires: SF-36, ChiQOL, sleeping questionnaires, IPAQ,QEWP, PCOS-QOL, Zung SAS, Zung SDS questionnaires, and the quantization table of TCM syndromes about PCOS will be used to assess the quality of life and sleep and the emotional state and to measure the moderate physical activity.

(9) Side effect profile.

(10) Expectation and credibility of treatment. 


\section{Data management and quality control of data}

Both the Case Record Form (CRF) and web-based electronic database will be used to manage individual participant data. Quality control of the data will be handled at two different levels: the investigators will be required to ensure the accuracy of the data as the first level of control when they input the records in CRF. The second level will include data monitoring and validation that will be carried out by an independent group. A web-based electronic database, ResMan (www.medresman.org), is used as a double input database. Finally, the individual participant data except the private information will be allowed to be share with the public within 6 months after the trial completion.

\section{Sample size calculations}

In our pilot study [25] evaluating the effect of acupuncture on insulin sensitivity in PCOS women with IR, HOMA-IR reduced significantly after 3 months of acupuncture treatment when an interim analysis was performed and changed from $(4.3 \pm 2.5)$ vs. $(3.7 \pm 2.1)$. HOMA-IR $\geq 2.14$ was considered to be abnormal. We anticipate that the HOMA-IR after 4 months of true acupuncture treatment will decrease $25 \%$, and sham acupuncture will be decreased $5 \%$ as it is well known that sham is not an inert procedure. Therefore, the difference is $20 \%$, with two-sided $\alpha$ assigned to be $5 \%$ and $\beta 20 \%$ at the upper limit, respectively, at which the power is $80 \%$ and the rate of attrition estimated to be $20 \%$. Thus, the sample size has been inflated from 95 to 114 per group, totaling 342 cases for the three arms. The sample size was calculated using the software statistics toolkit supported by the Department of Obstetrics and Gynecology of the Chinese University of Hong Kong (http://www.obg.cuhk.edu.hk/ResearchSupport/StatTools/index.php).

\section{Randomization and patient allocation}

Subjects will be randomly allocated into one of the three treatment arms by a 1:1:1 treatment ratio: (1) true acupuncture + metformin placebo, (2) sham acupuncture + metformin, and (3) sham acupuncture + metformin placebo. The Data Coordination Centre (DCC) statisticians will generate the randomization scheme for the study. The true and sham acupuncture treatments will be known only to the acupuncturists besides the DCC data manager. The metformin and placebo having the same package will be organized in a kit consisting of 4 sacks for each subject with 336 tablets in total, respectively. The kit will be labeled with an ID number mapped to the metformin and placebo known only to the DCC personnel. The metformin and placebo assignments will be double-blind to any site investigators.
A central randomization system will be used to allocate patients. Patients will be stratified based on three sites and randomly assigned. The Chinese Clinical Trial Registry takes the responsibility for generation of the random number sequence by computer software, and the allocation sequence is deposited in ResMan, a webbased database. When a new participant enrolls, the allocation information will be obtained by inputting a special password in ResMan. It is not possible to speculate the allocation status of the participant before getting the information.

\section{Statistical analysis strategy}

The Kolmogorov-Smirnov test will be used to test the normal distribution of continuous variables. Continuous variables will be presented by mean \pm standard deviation if they are normally distributed or by median with interquartile range if they are not normally distributed. Both per protocol (PP) analysis and intention-to-treat analysis (ITT) will be used to determine the robustness of the evidence. Analysis of variance (ANOVA) with Bonferroni corrections will be used for comparing the differences between groups of continuous data in accordance with a normal distribution or Kruskal-Wallis test for non-normal distribution data when appropriate; $\chi^{2}$ tests will be used for comparison of dichotomous data. For the primary outcome, analysis of covariance (ANCOVA) (accompanying LSMEAN and 95\% CI) will be used. ANOVA will be used to calculate the changes from baseline to after treatment and follow-up, respectively, and it will also be used to compare the differences between treatment arms with respect to the primary outcome, HOMA-IR. Effect of continuous data will be reported by both $P$-value and mean difference with 95\% confidence interval and categorical data by $P$-value and relative risk (RR) and risk difference (RD) with 95\% confidence interval; the number needed to treat (NNT) will be calculated if there is statistical significance between groups. All statistical analyses of the data will be performed by using SPSS software version 21.0 (SPSS Inc., Chicago, IL, USA).

\section{Discussion}

Although we have confirmed that the combination of manual and low frequency EA can improve insulin sensitivity, hormone levels, and anxiety situation in patients with PCOS by the prospective study (ClinicalTrials.gov NCT02026323) [25], it had the limitation of being a single center study without comparison groups. There is still a lack of high-quality studies to determine the effect of acupuncture on insulin sensitivity in PCOS women and IR.

Based on the results of the prospective observational study (ClinicalTrials.gov NCT02026323) [25], we designed this randomized, double-blind, placebo-controlled, and multi-center study protocol to evaluate whether 
acupuncture treatment is more effective than metformin and sham acupuncture in improving the insulin sensitivity of PCOS and IR. The use of a larger sample size in a randomized setting, controlled by sham acupuncture and common medicine of insulin-sensitizing agents, will reduce the risk of type II error. Therefore, the results of this study may provide evidence for using acupuncture in future clinical practice in this area.

\section{Trial status}

The study was registered at ClinicalTrials.gov on 24 June 2015. Recruitment was started on 11 November 2015 and is expected to end in March 2018. Recruitment is ongoing and 114 participants were randomized as of the end of December 2016. The final report will be available in 2018.

\section{Additional file}

Additional file 1: SPIRIT 2013 checklist. Recommended items to address in a clinical trial protocol and related documents. (PDF $106 \mathrm{~kb}$ )

\section{Acknowledgments}

The DSMB members include Mei Han (Beijing University of Chinese Medicine) and Min $\mathrm{Hu}$ (Goteborg University) chaired by Hongying Kuang at Heilongjiang University of Chinese Medicine.

\section{Funding}

This work was supported in part by the Natural Science Foundation of Guangdong Province in China (grant no. 2015A030310508) and funded by the Science and Technology Planning Project of Guangdong Province in China (grant no. 2014A020221060).

\section{Availability of data and materials}

Upon the completion of the study, supporting data will be available upon request.

\section{Authors' contributions}

$J L$ and EHYN contributed equally to this work. $J L$ drafted the manuscript for important intellectual content and sought funding and ethical approval. EHYN conceived and designed the study and critically revised the manuscript for important intellectual content. ESV critically revised the manuscript for important intellectual content and designed the acupuncture protocol. ZH, XS, HW, ML, CX, and NS had input to the design of the study and recruited subjects. ML helped to draft the manuscript and carried out acupuncture treatment. CY and JL carried out acupuncture treatment. TW was responsible for randomization and data management. HM conceived and designed the study and sought funding and ethical approval. All authors read and approved the final manuscript.

\section{Competing interests}

The authors declare that they have no competing interests.

\section{Consent for publication}

Not applicable.

\section{Ethics approval and consent to participate}

This study had been approved by the ethics committee of the First Affiliated Hospital of Guangzhou Medical University, Xuzhou Maternity \& Child Health Hospital, Dalian Municipal Women and Children's Medical Center, Guangdong Women and Children's Hospital, and Hexian Memorial Affiliated Hospital of Southern Medical University (Reference: 2015010). All participants signed the informed consent form prior to participation.

\section{Author details}

'Department of Traditional Chinese Medicine, the First Affiliated Hospital of Guangzhou Medical University, Guangzhou, China. ${ }^{2}$ Department of Obstetrics and Gynecology, the University of Hong Kong, Hong Kong Special Administrative Region, Hong Kong, China. ${ }^{3}$ Department of Physiology and Pharmacology, Karolinska Institute, Stockholm, Sweden. ${ }^{4}$ Department of Gynecology, Xuzhou Maternity \& Child Health Hospital, Xuzhou, China. ${ }^{5}$ Reproductive and Genetic Medical Center, Dalian Municipal Women and Children's Medical Center, Dalian, China. 'Department of Acupuncture and Moxibustion, Guangdong Provincial Hospital of Chinese Medicine, Guangzhou, China. ${ }^{7}$ Department of Reproductive Health and Infertility, Guangdong Women and Children Hospital, Guangzhou , China. ${ }^{8}$ Chinese Clinical Trial Registry, Beijing, China.

Received: 22 July 2016 Accepted: 17 February 2017

Published online: 09 March 2017

\section{References}

1. Lakkakula BV, Thangavelu M, Godla UR. Genetic variants associated with insulin signaling and glucose homeostasis in the pathogenesis of insulin resistance in polycystic ovary syndrome: a systematic review. J Assist Reprod Genet. 2013;30(7):883-95.

2. Katulski K, Czyzyk A, Podfigurna-Stopa A, Genazzani AR, et al. Pregnancy complications in polycystic ovary syndrome patients. Gynecol Endocrinol. 2014;30:1-5

3. Nestler JE. Role of hyperinsulinemia in the pathogenesis of the polycystic ovary syndrome, and its clinical implications. Semin Reprod Endocrinol. 1997;15(2):111-22.

4. Farrell K, Antoni MH. Insulin resistance, obesity, inflammation, and depression in polycystic ovary syndrome: biobehavioral mechanisms and interventions. Fertil Steril. 2010;94(5):1565-74.

5. Bhathena RK. Insulin resistance and the long-term consequences of polycystic ovary syndrome. J Obstet Gynaecol. 2011;31(2):105-10.

6. Hernandez-Valencia M, Hernandez-Rosas M, Zarate A. Care of insulin resistance in polycystic ovary syndrome. Ginecol Obstet Mex. 2010;78(11):612-6.

7. Legro RS, Dodson WC, Kunselman AR, et al. Benefit of delayed fertility therapy with preconception weight loss over immediate therapy in obese women with PCOS. J Clin Endocrinol Metab. 2016;12:jc20161659.

8. Franks S. When should an insulin sensitizing agent be used in the treatment of polycystic ovary syndrome? Clin Endocrinol (Oxf). 2011;74(2):148-51.

9. Pauli JM, Raja-Khan N, Wu X, et al. Current perspectives of insulin resistance and polycystic ovary syndrome. Diabet Med. 2011;28(12):1445-54.

10. Zhou G, Myers R, Li Y, et al. Role of AMP-activated protein kinase in mechanism of metformin action. J Clin Invest. 2001;108:1167-74.

11. Hwang KR, Choi YM, Kim JJ, et al. Effects of insulin-sensitizing agents and insulin resistance in women with polycystic ovary syndrome. Clin Exp Reprod Med. 2013;40(2):100-5.

12. Legro RS, Arslanian SA, Ehrmann DA, et al. Diagnosis and treatment of polycystic ovary syndrome: an Endocrine Society clinical practice guideline. J Clin Endocrinol Metab. 2013;98(12):4565-92.

13. Chang $C T$, Chen YC, Fang JT, et al. Metformin-associated lactic acidosis: case reports and literature review. J Nephrol. 2002;15(4):398-402.

14. Triggle $\mathrm{CR}$, Ding $\mathrm{H}$. Cardiovascular impact of drugs used in the treatment of diabetes. Ther Adv Chronic Dis. 2014;5(6):245-68.

15. Dunaif A. Drug insight: insulin-sensitizing drugs in the treatment of polycystic ovary syndrome-a reappraisal. Nat Clin Pract Endocrinol Metab. 2008;4(5):272-83.

16. Graham DJ, Ouellet-Hellstrom R, MaCurdy TE, et al. Risk of acute myocardial infarction, stroke, heart failure, and death in elderly Medicare patients treated with rosiglitazone or pioglitazone. JAMA. 2010;304(4):411-8.

17. Mamtani R, Haynes $K$, Bilker WB, et al. Association between longer therapy with thiazolidinediones and risk of bladder cancer: a cohort study. J Natl Cancer Inst. 2012;104(18):1411-21.

18. Betteridge DJ. Thiazolidinediones and fracture risk in patients with type 2 diabetes. Diabet Med. 2011;28(7):759-71.

19. Witt CM, Pach D, Brinkhaus B, et al. Safety of acupuncture: results of a prospective observational study with 229,230 patients and introduction of a medical information and consent form. Forsch Komplementmed. 2009;16(2):91-7.

20. Stener-Victorin E, Jedel E, Janson PO, et al. Low-frequency electroacupuncture and physical exercise decrease high muscle 
sympathetic nerve activity in polycystic ovary syndrome. Am J Physiol Regul Integr Comp Physiol. 2009;297(2):R387-95.

21. Manni L, Rocco ML, Barbaro Paparo S, et al. Electroacupucture and nerve growth factor: potential clinical applications. Arch Ital Biol. 2011;149(2):247-55.

22. Liang F, Koya D. Acupuncture: is it effective for treatment of insulin resistance? Diabetes Obes Metab. 2010;12(7):555-69.

23. Johansson J, Manneras-Holm L, Shao R, et al. Electrical vs manual acupuncture stimulation in a rat model of polycystic ovary syndrome: different effects on muscle and fat tissue insulin signaling. PLoS One. 2013;8(1):e54357.

24. Benrick A, Maliqueo M, Johansson J, et al. Enhanced insulin sensitivity and acute regulation of metabolic genes and signaling pathways after a single electrical or manual acupuncture session in female insulin-resistant rats. Acta Diabetol. 2014;51(6):963-72.

25. Yanhua Z, Stener-Victorin E, Ng HE, et al. How does acupuncture affect insulin sensitivity in women with polycystic ovary syndrome and insulin resistance? Study protocol of a prospective pilot study. BMJ Open. 2015;5(4):e007757.

26. Schulz KF, Altman DG, Moher D, for the CONSORT Group. CONSORT 2010 Statement: updated guidelines for reporting parallel group randomised trials. Trials. 2010:11:32. doi:10.1186/1745-6215-11-32.

27. Chan AW, Tetzlaff JM, Gotzsche PC, Altman DG, Mann H, Berlin JA, et al. SPIRIT 2013 explanation and elaboration: guidance for protocols of clinical trials. BMJ. 2013;346:e7586.

28. Zhao X, Ni R, Li L, et al. Defining hirsutism in Chinese women: a crosssectional study. Fertil Steril. 2011;96(3):792-6.

29. Ni RM, Mo Y, Chen $X$, et al. Low prevalence of the metabolic syndrome but high occurrence of various metabolic disorders in Chinese women with polycystic ovary syndrome. Eur J Endocrinol. 2009;161(3):411-8.

30. Chen $X$, Yang D, Li L, et al. Abnormal glucose tolerance in Chinese women with polycystic ovary syndrome. Hum Reprod. 2006;21(8):2027-32.

31. Vincent C, Lewith G. Placebo controls for acupuncture studies. J R Soc Med. 1995;88:199-202

32. MacPherson H, Altman DG, Hammerschlag R, et al. Revised Standards for Reporting Interventions in Clinical Trials of Acupuncture (STRICTA): extending the CONSORT statement. PLoS Med. 2010;7(6):e1000261.

33. Stener-Victorin E, Baghaei F, Holm G, et al. Effects of acupuncture and exercise on insulin sensitivity, adipose tissue characteristics, and markers of coagulation and fibrinolysis in women with polycystic ovary syndrome: secondary analyses of a randomized controlled trial. Fertil Steril. 2012;97:501-8.

34. Kuang H, Li Y, Wu X, et al. Acupuncture and clomiphene citrate for live birth in polycystic ovary syndrome: study design of a randomized controlled trila. Evid Based Complement Alternat Med. 2013;2013:527303.

35. McHorney CA, Ware Jr JE, Raczek AE. The MOS 36-Item Short-Form Health Survey (SF-36): II.Psychometric and clinical tests of validity in measuring physical and mental health constructs. Med Care. 1993;31(3):247-63.

36. Leung KF, Liu FB, Zhao L, et al. Development and validation of the Chinese Quality of Life Instrument. Health Qual Life Outcomes. 2005;3:26.

37. Buysse DJ, Reynolds 3rd CF, Monk TH, et al. The Pittsburgh Sleep Quality Index: a new instrument for psychiatric practice and research. Psychiatry Res. 1989;28(2):193-213.

38. Craig CL, Marshall AL, Sjostrom M, et al. International physical activity questionnaire: 12-country reliability and validity. Med Sci Sports Exerc. 2003;35(8):1381-95.

39. Borges MB, Morgan CM, Claudino AM, et al. Validation of the Portuguese version of the questionnaire on eating and weight patterns-revised (QEWP-R) for the screening of binge eating disorder. Rev Bras Psiquiatr. 2005;27(4):319-22.

40. Cronin L, Guyatt G, Griffith L, et al. Development of a health-related qualityof-life questionnaire (PCOSQ) for women with polycystic ovary syndrome (PCOS). J Clin Endocrinol Metab. 1998;83(6):1976-87.

41. Jiang M, Lu C, Zhang C, et al. Syndrome differentiation in modern research of traditional Chinesemedicine. J Ethnopharmacol. 2012;140(3):634-42.

42. Matthews DR, Hosker JP, Rudenski AS, et al. Homeostasis model assessment: insulin resistance and beta-cell function from fasting plasma glucose and insulin concentrations in man. Diabetologia. 1985;28(7):412-9.

43. Iwata M, Maeda S, Kamura Y, et al. Genetic risk score constructed using 14 susceptibility alleles for type 2 diabetes is associated with the early onset of diabetes and may predict the future requirement of insulin injections among Japanese individuals. Diabetes Care. 2012;35(8):1763-70.

44. Kahn SE, Prigeon RL, McCulloch DK, et al. Quantification of the relationship between insulin sensitivity and beta-cell function in human subjects. Evidence for a hyperbolic function. Diabetes. 1993;42(11):1663-72.

\section{Submit your next manuscript to BioMed Central and we will help you at every step:}

- We accept pre-submission inquiries

- Our selector tool helps you to find the most relevant journal

- We provide round the clock customer support

- Convenient online submission

- Thorough peer review

- Inclusion in PubMed and all major indexing services

- Maximum visibility for your research

Submit your manuscript at www.biomedcentral.com/submit
( ) BioMed Central 